\title{
Enhancing the energy of terahertz radiation from plasma produced by intense femtosecond laser pulses
}

\section{$\operatorname{AUTHOR}(S):$}

Jahangiri, Fazel; Hashida, Masaki; Tokita, Shigeki; Nagashima, Takeshi; Hangyo, Masanori; Sakabe, Shuji

\section{CITATION:}

Jahangiri, Fazel ... [et al]. Enhancing the energy of terahertz radiation from plasma produced by intense femtosecond laser pulses. Applied Physics Letters 2013, 102(19): 191106.

\section{ISSUE DATE:}

2013-05

URL:

http://hdl.handle.net/2433/174313

RIGHT:

(C) 2013 AIP Publishing LLC 


\section{AIP Applied physises \\ Letters}

\section{Enhancing the energy of terahertz radiation from plasma produced by intense femtosecond laser pulses}

Fazel Jahangiri, Masaki Hashida, Shigeki Tokita, Takeshi Nagashima, Masanori Hangyo et al.

Citation: Appl. Phys. Lett. 102, 191106 (2013); doi: 10.1063/1.4804582

View online: http://dx.doi.org/10.1063/1.4804582

View Table of Contents: http://apl.aip.org/resource/1/APPLAB/v102/i19

Published by the American Institute of Physics.

Additional information on Appl. Phys. Lett.

Journal Homepage: http://apl.aip.org/

Journal Information: http://apl.aip.org/about/about_the_journal

Top downloads: http://apl.aip.org/features/most_downloaded

Information for Authors: http://apl.aip.org/authors

\section{ADVERTISEMENT}

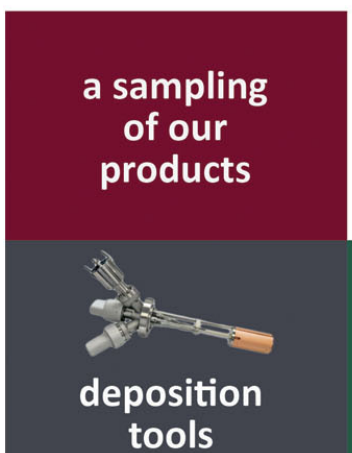

tools

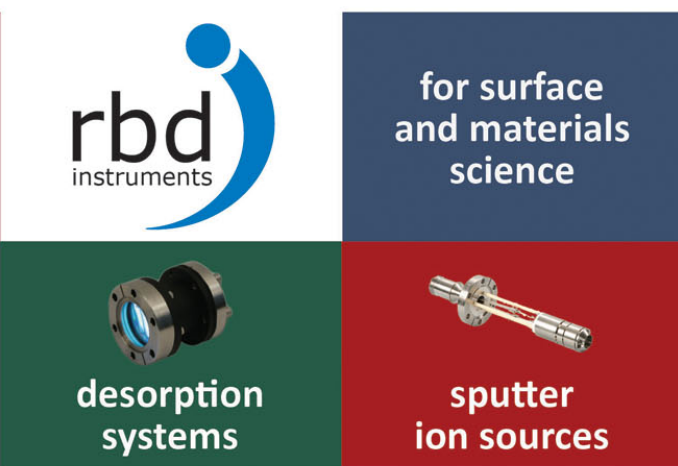

systems

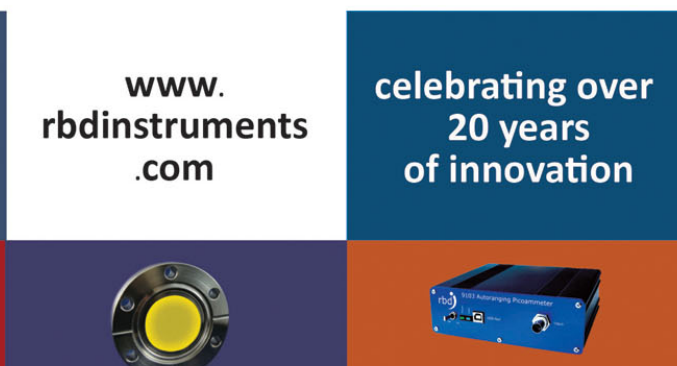

usb
ion sources
viewports
picoammeters 


\title{
Enhancing the energy of terahertz radiation from plasma produced by intense femtosecond laser pulses
}

\author{
Fazel Jahangiri, ${ }^{1,2, a)}$ Masaki Hashida, ${ }^{1,3, a)}$ Shigeki Tokita, ${ }^{1,3}$ Takeshi Nagashima, ${ }^{3,4}$ \\ Masanori Hangyo, ${ }^{3,4}$ and Shuji Sakabe ${ }^{1,3}$ \\ ${ }^{1}$ Advanced Research Center for Beam Science, ICR, Kyoto University, Kyoto, Japan \\ ${ }^{2}$ Laser and Plasma Research Institute, Shahid Beheshti University, Tehran, Iran \\ ${ }^{3}$ Department of Physics, GSS, Kyoto University, Kyoto, Japan \\ ${ }^{4}$ Institute of Laser Engineering, Osaka University, Osaka, Japan
}

(Received 12 February 2013; accepted 24 April 2013; published online 13 May 2013)

\begin{abstract}
Terahertz $(\mathrm{THz})$ radiation from atomic clusters illuminated by intense femtosecond laser pulses is investigated. By studying the angular distribution, polarization properties and energy dependence of $\mathrm{THz}$ waves, we aim to obtain a proper understanding of the mechanism of $\mathrm{THz}$ generation. The properties of $\mathrm{THz}$ waves measured in this study differ from those predicted by previously proposed mechanisms. To interpret these properties qualitatively, we propose that the radiation is generated by time-varying quadrupoles, which are produced by the ponderomotive force of the laser pulse. (C) 2013 AIP Publishing LLC. [http://dx.doi.org/10.1063/1.4804582]
\end{abstract}

The application of $\mathrm{THz}$ radiation is expanding in remote sensing, imaging, spectroscopic analyses, and other promising areas. As a promising source for $\mathrm{THz}$ radiation, plasma induced by femtosecond laser pulses has been extensively studied. ${ }^{1-7}$ Comparison of different gas and solid targets has revealed that solid targets are more efficient than gas targets, ${ }^{8-12}$ owing to their much higher laser absorption. ${ }^{13}$ However, considerable $\mathrm{THz}$ reflection at the vacuum-plasma interface and generation of a large amount of debris are significant problems associated with $\mathrm{THz}$ radiation produced from solid targets. We have recently proposed atomic clusters as an alternative target for the production of $\mathrm{THz}$ radiation. ${ }^{9,14}$ These combine advantages of both gas and solid targets: laser absorption is higher than for gases, resulting in stronger $\mathrm{THz}$ emission, and there is much less debris generation than for solid targets. We have reported that the plasma of argon clusters as well-isolated solid targets can generate $\mathrm{THz}$ pulses with energies two orders of magnitude higher than argon gas, distributed in a scissors-like shape in the horizontal plane. ${ }^{14}$ Although, this improvement can be directly ascribed to higher laser absorption in atomic clusters, ${ }^{15,16}$ a deep understanding of the physical mechanism is still desirable in order to explain the overall characteristics of $\mathrm{THz}$ radiation. Precise measurements of $\mathrm{THz}$ power in both horizontal and vertical planes around the plasma together with polarization analyses are presented here to inform the discussion about the physical mechanism of $\mathrm{THz}$ generation. The measurements reveal a conical angular distribution, in both forward and backward directions, with radial polarization and also a significant emission in the forward direction with elliptical polarization. The total $\mathrm{THz}$ power has a square dependence on laser pulse energy. These results are not consistent with previously proposed mechanisms, including electron oscillations driven by the laser wake field, ${ }^{1,2}$ nonlinear frequency mixing processes in air plasmas ${ }^{4-8}$ and transition radiation from relativistic electrons induced by the laser

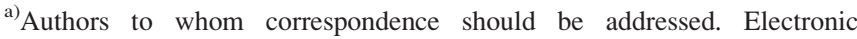
addresses: f_jahangiri@sbu.ac.ir and hashida@laser.kuicr.kyoto-u.ac.jp
}

wake field passing through the plasma-vacuum boundary. ${ }^{11,12}$ Based on the observed properties, a qualitative mechanism is proposed and discussed.

Argon clusters were produced by injection of high pressure argon gas $(<8 \mathrm{MPa})$ into a high-vacuum chamber, through a conical nozzle. High-energy pulses $(<80 \mathrm{~mJ})$ from a Ti:sapphire laser ${ }^{17}$ with a center wavelength of $800 \mathrm{~nm}$ and a duration of $130 \mathrm{fs}$ were focused onto the argon cluster beam by a plano-convex lens with a focal length of $200 \mathrm{~mm}$. The gas injection was synchronized with the laser pulses for maximum laser energy absorption (details can be found in Ref. 18). The vacuum chamber was made of fused silica with a transparency of $\sim 80 \%$ for $\mathrm{THz}$ waves at frequencies of $<3 \mathrm{THz} .{ }^{14}$ The experiment used the same setup as described in Ref. 14. The mean size of the clusters, which depends on the backing pressure, was determined by the Rayleigh scattering method ${ }^{19}$ to be $\sim 10^{4}$ atoms at $7 \mathrm{MPa}$. This corresponds to a mean cluster diameter of $\sim 10 \mathrm{~nm} .^{20}$ Considering the atomic density of argon gas flowing at backing pressure of $7 \mathrm{MPa}$ that is estimated to be $\sim 10^{17} \mathrm{~cm}^{-3}$ (Ref. 21) and also considering the mean size of the clusters ( $\sim 10^{4}$ atoms), the average density would be equivalent to 10 cluster $/ \mu \mathrm{m}^{3}$ and the mean distance between adjacent clusters is estimated to be of the order of $\sim 1 \mu \mathrm{m}$. The laser spot diameter was $\sim 17 \mu \mathrm{m}$. A schematic diagram of our experimental setup is shown in Fig. 1(a). The laser pulses were linearly polarized in the horizontal direction, parallel to the optical table. Argon clusters were irradiated by the laser pulses, and the high-energy ions generated by Coulomb explosion of the clusters were detected by a time-of-flight measurement. ${ }^{18,19}$ The energy distribution of the generated ions was used to confirm the production of the clusters. The $\mathrm{THz}$ waves produced from the interaction of laser pulses with the cluster beam were collimated by a polyethylene lens with focal length of $150 \mathrm{~mm}$, which was placed outside the vacuum chamber. After passing through a wire grid polarizer, the $\mathrm{THz}$ waves were focused onto a liquid-He-cooled $\mathrm{InSb}$ bolometer by an off-axis parabolic mirror. A time interferogram of $\mathrm{THz}$ pulses was obtained by using a 
(a)

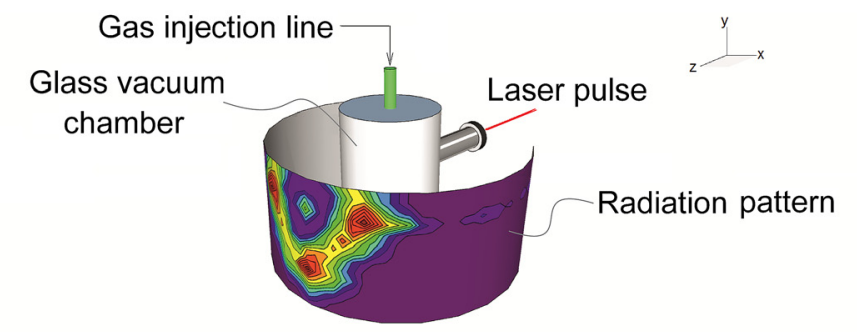

(b)

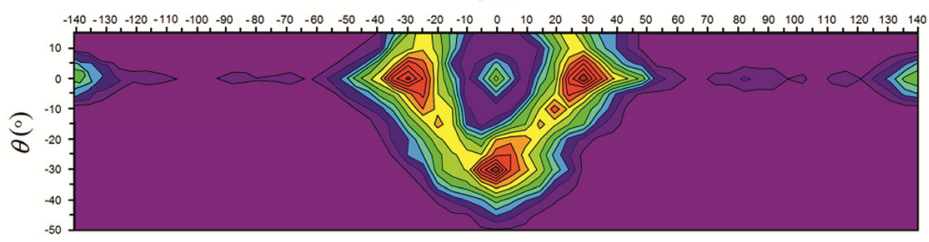

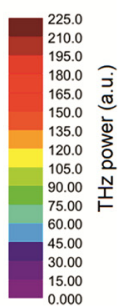

FIG. 1. (a) Schematic of experimental setup with (b) the $2 \mathrm{D}$ angular distribution of $\mathrm{THz}$ waves. The horizontal and vertical measurement angles were limited by the laser focusing setup and the gas injection line, respectively.
Martin-Puplett interferometer and the bolometer. The details of these measurements have been presented in Ref. 14. The spectrum of $\mathrm{THz}$ pulses was found by performing an inverse Fourier transform; the spectrum does not strongly depend on laser energy or backing pressure. ${ }^{14}$ A thick plate of polystyrene foam and a black polyethylene sheet were placed in front of the bolometer to block unwanted infrared and visible light emitted or scattered by the clusters.

The angular distribution of $\mathrm{THz}$ waves measured in two dimensions at a backing pressure of $7 \mathrm{MPa}$ and a laser energy of $30 \mathrm{~mJ}$ shows that $\mathrm{THz}$ waves had a conical distribution in both the forward and backward directions (Fig. 1(b)). By moving the bolometer along a circular path around the chamber, THz power was measured in the horizontal $(\phi)$ and vertical $(\theta)$ directions at step sizes of $\Delta \phi=5^{\circ}$ and $\Delta \theta=5^{\circ}$. The reference angle was chosen such that the laser propagation direction was $(\phi, \theta)=(0,0)$. This figure also shows that the radiation cone includes significant peaks, detected in planes containing the angles $\phi=0$ and $\theta=0$. Furthermore, considerable radiation was observed in the direction of laser propagation.

Using a wire grid polarizer, the horizontal and vertical components of the $\mathrm{THz}$ waves were separately measured at different angles. Comparing the horizontal and vertical components gives the polarization direction (see Fig. 2). The observed polarization pattern was reminiscent of that expected

$$
\phi\left({ }^{\circ}\right)
$$

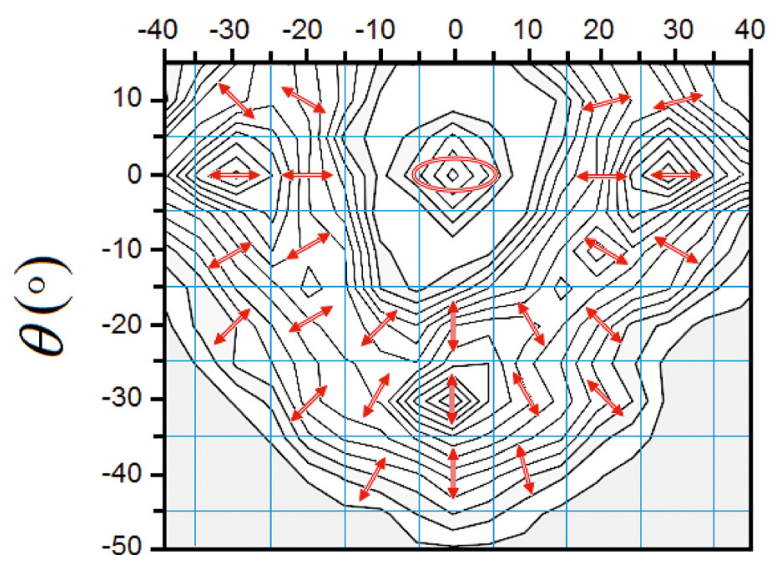

FIG. 2. Radial and elliptical polarization of $\mathrm{THz}$ waves observed on the radiation cone and on the laser propagation axis, respectively. for a radial polarization. By inserting a half-waveplate in front of the focusing lens, we confirmed that the polarization of the $\mathrm{THz}$ waves was independent of laser polarization. Our measurements show that the considerable radiation observed in the direction of laser propagation cannot be a conical beam. The polarizations of $\mathrm{THz}$ waves were measured before and after passing through a quarter-waveplate designed for a frequency of $0.5 \mathrm{THz}$ (WPCQD47-OW600L/4, Tydex Co.). Bandpass filters (BPF0.5-47, Tydex Co.), which were inserted in the path of the $\mathrm{THz}$ waves, passed the frequencies specified by the quarter-waveplates. The polarization of the THz waves after passing through the quarter-waveplate was almost linear, which shows that the initial $\mathrm{THz}$ polarization in this direction was elliptical with a measured ellipticity of $\sim 0.5$ (see Fig. 3). The elliptical polarization of $\mathrm{THz}$ waves generated along the laser axis is consistent with a four-wave mixing process. ${ }^{14}$

The measured properties of the $\mathrm{THz}$ waves differed from those expected from previously proposed mechanisms. ${ }^{1,2,11,12,22,23}$ The nonlinear current associated with laser intensity inside the plasma ${ }^{1,2}$ predicts a polarization dependent on laser polarization. However, we observed that the polarization state of the $\mathrm{THz}$ wave distributed through the cones was independent of laser polarization. This suggests that the contribution from the nonlinear current was negligible.

It has also been proposed that transition radiation generated by laser-wakefield-accelerated electron bunches passing through a plasma-vacuum boundary can result in $\mathrm{THz}$ radiation. ${ }^{11,12}$ According to the theory, ${ }^{24}$ when the plasma is assumed to be a perfect conductor, the angular energy distribution of radiation from a single electron traversing the plasma-vacuum boundary is given by

$$
W\left(\omega_{T H z}, \Theta\right) \propto \frac{\beta^{2} \sin ^{2} \Theta}{\left(1-\beta^{2} \cos ^{2} \Theta\right)^{2}},
$$

where $\Theta$ is measured with respect to the laser pulse propagation direction and $\beta=v / c$ ( $v$ : electron velocity; $c$ : speed of light in a vacuum). For laser intensities of $10^{17} \mathrm{~W} / \mathrm{cm}^{2}$, such as those used in the present work, the ponderomotive energy $K=e^{2} E^{2} /\left(m \omega^{2}\right)$ is $\sim 6 \mathrm{keV},{ }^{25}$ where $e$ is the electron charge, $E$ is the laser electric field, $m$ is the electron mass, and $\omega$ is laser frequency. This corresponds to an electron velocity of $\beta \approx 0.15$. For these parameters, it is expected from Eq. (1) 
(a)

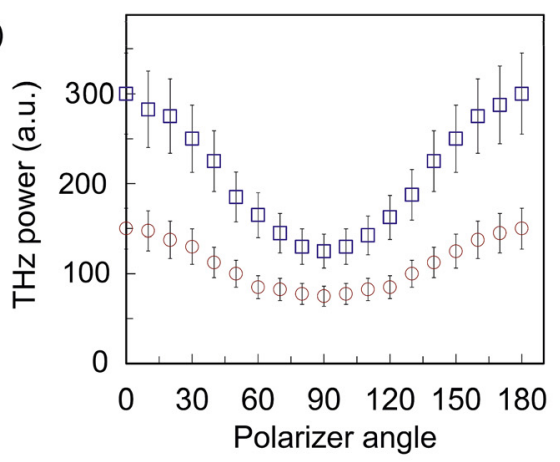

(b)

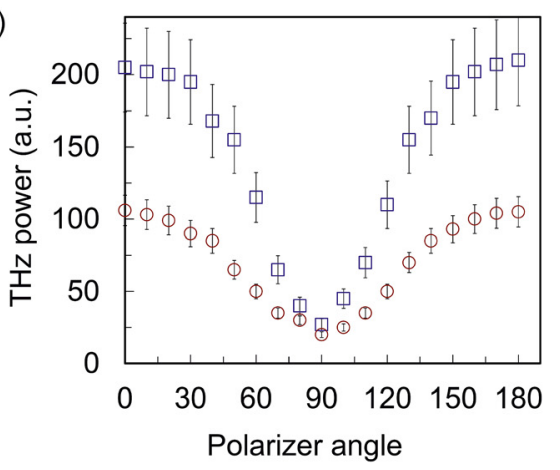

FIG. 3. Polarization analyses of THz radiation along the laser propagation axis (a) before and (b) after passing through a quarter-waveplate, at laser energies of $10 \mathrm{~mJ}$ (circle) and $40 \mathrm{~mJ}$ (square). that the angular distribution of $\mathrm{THz}$ wave power caused by the transition radiation should exhibit a maximum in the direction of $\Theta \approx 90^{\circ}$, which is inconsistent with the measured angular distribution. Therefore, we conclude that here transition radiation can be ruled out as a dominant contributor to the production of $\mathrm{THz}$ radiation.

There is also the possibility that the $\mathrm{THz}$ radiation was Cherenkov radiation caused by a high-energy electron beam. $^{22,23}$ The real part $n_{\mathrm{THz}}$ of the refractive index of the plasma at $0.5 \mathrm{THz}$ is estimated to be $\sim 40$, under the Drude model with an electron scattering time of $\sim 0.01 \mathrm{ps}$. This value comes from the fact that, for an assumed electron density of $\sim 10^{18} \mathrm{~cm}^{-3}$, the electrons would be scattered by the clusters within a scattering time of $\tau=d /(c \beta)$, where $d$ is the mean distance between the clusters. Then, the Cherenkov radiation would be emitted in a direction of $\cos ^{-1}\left(1 / \beta n_{\mathrm{THz}}\right)$ $\approx 80^{\circ}$, if the boundary effect is ignored. If the $\mathrm{THz}$ radiation in direction of $30^{\circ}$ (half-cone angle) were Cherenkov radiation, the value of $\beta$ would be $\sim 0.029$. This value is too small for the laser intensity we used; therefore, Cherenkov radiation cannot be a major factor. Moreover, this model cannot explain the backward $\mathrm{THz}$ radiation observed from the argon cluster plasma.

To interpret the angular distribution and polarization of $\mathrm{THz}$ waves, we propose a model based on radiation from time-varying electric quadrupoles ${ }^{26,27}$ generated by electrons moving in response to the ponderomotive force of the laser pulses. The electrons in the vicinity of the laser pulse are expelled to the outside of the laser-produced plasma by the ponderomotive force originating from the radial gradient of the electric field. This motion of the electrons produces a core of positive charge surrounded by symmetrically distributed electrons. Since we did not apply an external field, the plasma was axially symmetric with zero transverse dipole moment. ${ }^{28}$ Such plasma volumes can be theoretically considered as a simple radiating quadrupole source. ${ }^{26}$ An axial electric quadrupole is formed by the distribution of charges on a line with two ions $(+2 q)$ at the midpoint and two electrons $(-q)$ located symmetrically around it, as shown schematically in Fig. 4. The axial quadrupoles prefer to align parallel to the directions of laser propagation, laser polarization, and gas injection. As the laser pulse passes through the plasma, the quadrupoles at each point in the plasma oscillate with a period of $\sim 130 \mathrm{fs}$, which is the duration of the laser pulse. Therefore, waves radiated from the quadrupoles have a spectrum in the $\mathrm{THz}$ frequency region. Nonresonant $\mathrm{THz}$ radiation and scattering caused by the electrons may result in a broadening of the spectrum around the central wavelength. According to our measurements, the wavelength of $\mathrm{THz}$ radiation is $\geq 300 \mu \mathrm{m}$, much smaller than the plasma dimensions, which was estimated from images of fluorescent light scattered from the plasma. ${ }^{14}$ Therefore, the total radiation from plasma is over the sum of the radiation coming from each small region containing the electric axial quadrupoles. According to electromagnetic field theory, the angular distribution of radiated power from an axial quadrupole is a four-lobed pattern ${ }^{26,29}$

$$
\frac{d P}{d \Omega} \propto \sin ^{2} \theta \cos ^{2} \theta
$$

where $\theta$ is the angle between the observation point and the axis of the quadrupole. Therefore, THz radiation from quadrupoles oscillating in a defined direction is conically distributed around that axis in the forward and backward directions.

The local density of argon clusters is much higher than the density of the argon gas just after exiting the nozzle of injection system. ${ }^{14,15}$ Since the ponderomotive energy is proportional to the plasma density, it will be much higher inside the argon cluster plasma than in a gas plasma, resulting in much stronger quadrupole radiation. The laser absorption $\left(A_{\text {cluster }}\right)$ of an argon cluster has been measured to be $\sim 30$-fold that of argon gas, at the same atomic density and same laser energy. It has been found that $\mathrm{THz}$ radiation and laser absorption of argon clusters have a square $\left(I_{\mathrm{THz}} \propto P^{2}\right)$ and linear dependence $\left(A_{\text {cluster }} \propto P\right)$ on backing pressure $(P)$, respectively; resulting in $I_{\mathrm{THz}} \propto\left(A_{\text {cluster }}\right)^{2}$. Therefore, a 900 -fold enhancement might be expected in $\mathrm{THz}$ power when a 30-fold increase is observed in laser absorption. The slightly smaller experimentally observed enhancement (600-fold $)^{14}$ might be attributed to the saturation of $\mathrm{THz}$ power at high backing pressures. ${ }^{9}$ Furthermore, since the quadrupoles are induced by ponderomotive forces within the plasma, a square dependence on laser energy is expected for THz radiation. ${ }^{14,27}$ This is consistent with our observations,

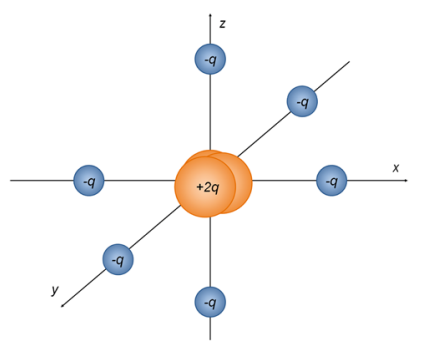

FIG. 4. Configuration of electric quadrupoles in the plasma, parallel and perpendicular to the laser propagation direction $(z)$. The perpendicular directions are the directions of the laser polarization (x) and gas jet injection (y). 


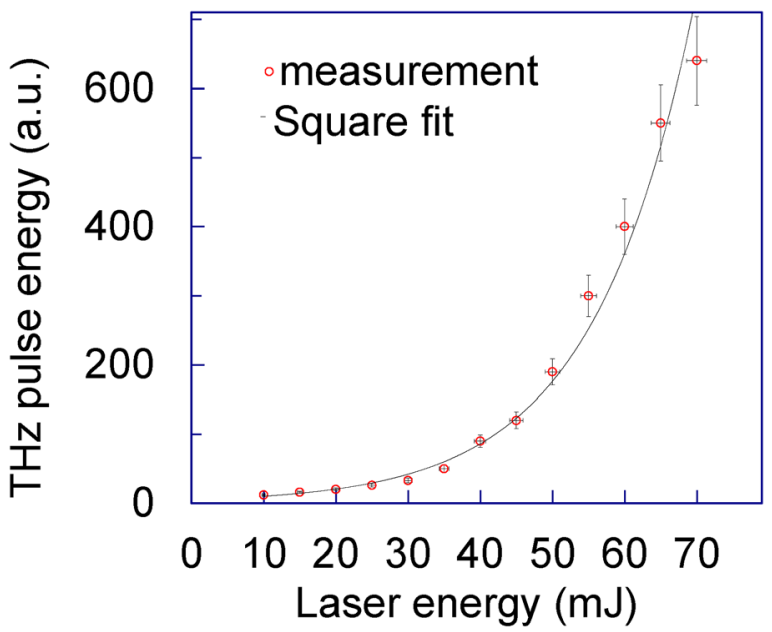

FIG. 5. The energy of the THz radiation has a square dependence on laser energy.

as shown in Fig. 5. It is worth pointing out that, due to technical limitations, the laser energy was kept less than $80 \mathrm{~mJ}$, so we did not observe any saturation at high laser energies. The non-saturated $\mathrm{THz}$ power at high laser energies reveals the potential for the extraction of higher $\mathrm{THz}$ energies from argon cluster plasma.

In addition to the conical distributions, four significant peaks were also observed in the directions of $(\theta, \varphi)=\left(0^{\circ}, 30^{\circ}\right)$ and $\left(-30^{\circ}, 0^{\circ}\right)$, as shown in Fig. 1(b). It seems that quadrupole radiation can also explain the appearance of these peaks. As discussed above, the quadrupoles are generated parallel and transverse to the laser propagation direction. Just as the parallel quadrupoles generate $\mathrm{THz}$ waves in forward and backward cones aligned along the direction of laser propagation ( $z$ direction), the transverse quadrupoles can generate $\mathrm{THz}$ radiation in cones with axes in the $x$ and $y$ directions. In agreement with previous reports, ${ }^{23}$ we have recently shown ${ }^{14}$ that the opening angle of the radiation cones is expected to be proportional to $\left(\lambda / d_{i}\right)^{0.5}$, where $\lambda$ is the wavelength of radiation and $d_{i}$ is the length of the plasma along the radiation direction $(i=x, y$, or $z)$. Assuming that the plasma is cylindrical along $z$ direction with length of $d$ and radius of $R$, plasma lengths are $d_{x}=R, d_{y}=R$, and $d_{z}=d$. Experimental measurements of plasma dimensions show that the radius of the plasma, $R$, is around a quarter of its length, $d$ (i.e., $d_{x}=d_{y}=1 / 4 d_{z}$ ). Thus the opening angle of the radiation cones in the $x$ and $y$ directions should be $\sim 2$-fold larger than that observed in the $z$ direction $\left(30^{\circ}\right)$, that is, $\sim 60^{\circ}$. Therefore, the radiation cones with axes along the $x$ and $y$ directions are, respectively, superposed at angles of $(\theta, \varphi)=\left(0^{\circ}\right.$, $\left.30^{\circ}\right)$ and $\left(-30^{\circ}, 0^{\circ}\right)$ on the radiation cones along the $\mathrm{z}$ direction, exactly where the four observed peaks appear.

In summary, the polarization properties, angular distribution, and laser energy dependence of $\mathrm{THz}$ waves generated from argon cluster plasma have been studied and compared with the properties expected if they were produced by previously proposed mechanisms. Differences between the expected and observed properties allow us to reject all previously proposed models for the generation of $\mathrm{THz}$ radiation. We propose that the $\mathrm{THz}$ radiation is generated by electric quadrupoles. This model can explain the polarization properties, energy dependence, and backing pressure dependence of $\mathrm{THz}$ waves.

This work was supported by a Grant-in-Aid for Scientific Research (Grant No. 23226002), by a Grant-in-Aid for the GCOE Program "The Next Generation of Physics, Spun from Universality and Emergence" from the Ministry of Education, Culture, Sports, Science and Technology of Japan, by the Collaborative Research Program of the Institute for Chemical Research, Kyoto University (Grant No. 2011-52), and by the joint research project of the Institute of Laser Engineering, Osaka University (under the proposal "Development of diagnostics of laser produced plasma by terahertz emission spectroscopy").

${ }^{1}$ H. Hamster, A. Sullivan, S. Gordon, W. White, and R. W. Falcone, Phys. Rev. Lett. 71, 2725 (1993).

${ }^{2}$ H. Hamster, A. Sullivan, S. Gordon, and R. W. Falcone, Phys. Rev. E 49, 671 (1994).

${ }^{3}$ T. Loffler and H. G. Roskos, J. Appl. Phys. 91, 2611 (2002).

${ }^{4}$ F. Jahangiri, M. Hashida, S. Tokita, T. Nagashima, M. Hangyo, and S. Sakabe, Appl. Phys. Lett. 99, 161505 (2011).

${ }^{5}$ D. J. Cook and R. M. Hochstrasser, Opt. Lett. 25, 1210 (2000).

${ }^{6}$ X. Xie, J. Dai, and X.-C. Zhang, Phys. Rev. Lett. 96, 075005 (2006).

${ }^{7}$ T. J. Wang, J. F. Daigle, S. Yuan, F. Theberge, M. Chateauneuf, J. Dubois, G. Roy, H. Zeng, and S. L. Chin, Phys. Rev. A 83, 053801 (2011).

${ }^{8}$ G. Rodriguez and G. L. Dakovski, Opt. Express 18, 15130 (2010).

${ }^{9}$ T. Nagashima, H. Hirayama, K. Shibuya, M. Hangyo, M. Hashida, S. Tokita, and S. Sakabe, Opt. Express 17, 8807 (2009).

${ }^{10}$ N. Yugami, K. Ninomiya, K. Kobayashi, and H. Noda, Jpn. J. Appl. Phys. 45, L1051 (2006).

${ }^{11}$ W. P. Leemans, E. Esarey, J. van Tilborg, P. A. Michel, C. B. Schroeder, C. Tóth, C. G. R. Geddes, and B. A. Shadwick, IEEE Trans. Plasma Sci. 33, 8 (2005)

${ }^{12}$ J. van Tilborg, C. B. Schroeder, C. V. Filip, Cs. Tóth, C. G. R. Geddes, G. Fubiani, R. Huber, R. A. Kaindl, E. Esarey, and W. P. Leemans, Phys. Rev. Lett. 96, 014801 (2006).

${ }^{13}$ D. F. Price, R. M. More, R. S. Walling, G. Guethlein, R. L. Shepherd, R. E. Stewart, and W. E. White, Phys. Rev. Lett. 75, 252 (1995).

${ }^{14}$ F. Jahangiri, M. Hashida, T. Nagashima, S. Tokita, M. Hangyo, and S. Sakabe, Appl. Phys. Lett. 99, 261503 (2011).

${ }^{15}$ T. Ditmire, T. Donnelly, A. M. Rubenchik, R. W. Falcone, and M. D. Perry, Phys. Rev. A 53, 3379 (1996).

${ }^{16}$ J. Zweiback, T. Ditmire, and M. D. Perry, Phys. Rev. A 59, R3166 (1999).

${ }^{17}$ S. Tokita, M. Hashida, S. Masuno, S. Namba, and S. Sakabe, Opt. Express 16, 14875 (2008).

${ }^{18}$ S. Sakabe, K. Shirai, M. Hashida, S. Shimizu, and S. Masuno, Phys. Rev. A 74, 043205 (2006).

${ }^{19}$ M. Hirokane, S. Shimizu, M. Hashida, S. Okada, S. Okihara, F. Sato, T. Iida, and S. Sakabe, Phys. Rev. A 69, 063201 (2004).

${ }^{20}$ O. F. Hagena, Rev. Sci. Instrum. 63, 2374 (1992).

${ }^{21}$ O. F. Hagena, Surf. Sci. 106, 101 (1981).

${ }^{22}$ T. Takahashi, Y. Shibata, K. Ishi, M. Ikegami, M. Oyamada, and Y. Kondo, Phys. Rev. E 62, 8606 (2000).

${ }^{23}$ C. D'Amico, A. Houard, S. Akturk, Y. Liu, J. L. Bloas, M. Franco, B. Prade, A. Couairon, V. T. Tikhonchuk, and A. Mysyrowicz, New J. Phys. 10, 013015 (2008)

${ }^{24}$ U. Happek, A. J. Sievers, and E. B. Blum, Phys. Rev. Lett. 67, 2962 (1991).

${ }^{25}$ R. W. Boyd, Nonlinear Optics, 2nd ed. (Academic Press, New York, 2003), Chap. 13.

${ }^{26}$ J. D. Jackson, Classical Electrodynamics, 3rd ed. (John Wiley \& Sons, New York, 1998).

${ }^{27}$ N. A. Panov, O. G. Kosareva, V. A. Andreeva, A. B. Savel'ev, D. S. Uryupina, R. V. Volkov, V. A. Makarov, and A. P. Shkurinov, JETP Lett. 93, 638 (2011).

${ }^{28}$ S. A. Hosseini, Q. Luo, B. Ferland, W. Liu, S. L. Chin, O. G. Kosareva, N. A. Panov, N. Akozbek, and V. P. Kandidov, Phys. Rev. A 70, 033802 (2004).

${ }^{29}$ L. D. Landau and E. M. Lifshitz, Course of Theoretical Physics: The Classical Theory of Fields (Pergamon, 1971), Vol. 2. 